\title{
Hypotonic Cerebral Palsy
}

National Cancer Institute

\section{Source}

National Cancer Institute. Hypotonic Cerebral Palsy. NCI Thesaurus. Code C116906.

A type of cerebral palsy characterized by decreased muscle tone. 